\title{
DEEP RAINRATE ESTIMATION FROM HIGHLY ATTENUATED DOWNLINK SIGNALS OF GROUND-BASED COMMUNICATIONS SATELLITE TERMINALS
}

\author{
Kumar Vijay Mishra, Bhavani Shankar M. R. and Björn Ottersten \\ Interdisciplinary Centre for Security, Reliability and Trust (SnT), University of Luxembourg, Luxembourg
}

\begin{abstract}
While the use of weather radars to continuously monitor the spatiotemporal dynamics of precipitation has grown in recent years, these systems are expensive and sparsely deployed across the world. In this context, densely located ground-based terminals for interactive satellite services have the potential for dual-use as weather sensors because they measure rain-attenuated power of the downlink signal. Although in the millimeter-wave regime, the rain rate has almost a linear relationship with specific attenuation, lack of other weather radar observables at satellite terminals imposes a daunting task of extracting rainfall rate from these highly attenuated signals. We address this problem by designing a deep convolutional neural network (CNN) that learns the relationship between the signal attenuation and rainfall rate observed by weather radars and rain gauges at a given location. During the prediction stage, the CNN accepts downlink attenuation as input and classifies the rain intensity which is then used to apply an appropriate rainfall estimator. Our experiments with real data show that, despite severe attenuation, CNN-based downlink rainfall accumulations closely follow the nearest C-band German weather service Deutscher Wetterdienst (DWD) radar.
\end{abstract}

Index Terms - Convolutional neural network, rainfall estimation, signal attenuation, satellite communication, weather radar

\section{INTRODUCTION}

In recent years, global environment change has become a worldwide concern $[1,2]$. During past few decades, national weather radar networks have merged as powerful and essential means to monitor space-time variability of precipitation in developed countries [3, 4]. Although rainfall estimation based on scanning weather radar measurements has been increasingly adopted, it is well-known that such estimates suffer from large uncertainties [5] and anomalous signal propagation [6]. Further, considerably large portions of the world, especially developing countries, are unable to afford dense deployment of state-of-the-art weather radars. Existing weather radar networks in the developed world are restricted by their large footprint and cost to cover lower troposphere at high spatio-temporal resolutions [3, 7]. For instance, the NEXRAD network of the U. S. National Weather Service is able to yield rainfall estimates at only $\sim 1-4 \mathrm{~km}^{2}$ resolution for a 14-minute volume scan.

In this context, alternative low-cost, in situ weather sensors such as rain gauges and disdrometers provide valuable high-resolution characterization of precipitation structure [7, 8]. Recently, opportunistic dual-use of communication satellite links as rainfall sensing devices has gained much attention in regions where coverage of ground-based weather radars is unavailable [9-12]. Conventional S- and C-band weather radars are very expensive but there are millions of ground

This work was partially funded by the Luxembourg National Research Fund (FNR) for the Proof of Concept project RAFAEL bearing contract $\mathrm{POC} / 17 / 11648950$. satellite terminals already spread across the world. Although these stations are primarily intended for interactive satellite services, they possess capability to measure the rain-induced attenuation of the downlink signal and relaying this information to a central location (gateway station) in real-time. By correlating these downlink signals with colocated vertical profile measurements obtained by atmospheric sounders, valuable precipitation information is obtained. While both communications satellites and spaceborne weather radars provide measurements from cloud-tops to the ground, the former far outnumber existing space weather radars such as NASA's Tropical Rainfall Measurement Mission (TRMM) and Global Precipitation Measurement (GPM) [13]. There is, therefore, immense recent interest in exploiting these signals of opportunity for rainfall estimation [12]. Note that this is different than recent works on harnessing rainfall from commercial microwave links (CML) for cellular communication [14] which do not propagate over the cloud-tops.

Communication satellites operate at lower millimeter-wave (mmWave) bands (upward from $30 \mathrm{GHz}$ ) because of the availability of wideband spectrum and small size of antennas at these frequencies. Ground-based weather radars typically avoid transmission at these frequencies because of very high attenuation of transmit signal due to propagation in the rain medium $[15,16]$. Further, Mie scattering effects are significant at mm-Wave thereby causing difficulties in the computation of scattering amplitudes [17-19]. Nonetheless, when S- and C-band weather radars are unavailable, a few studies have yielded valuable but less accurate rainfall estimates using mm-Wave weather radars [20]. Further, mm-Wave is helpful in detecting small hydrometeors such as graupel, cloud particles, drizzle and light snow [21]. Recently, it has been demonstrated that short-range rain rate retrieval is possible with mm-Wave vertical-pointing radars [22].

The mm-Wave precipitation estimation algorithms exploit the fact that both rain rate $R$ and the range derivative of attenuation (or specific attenuation) $A$ are approximately proportional to the 3.65 th rain drop size distributions (DSD) moment [23]. This results in a nearly linear relationship between $R$ and $A$. Moreover, the fact that this connection is relatively immune, $\sim 10 \%$, to variability in temperature and DSDs is entirely fortuitous [20].

However, direct application of this linear $R-A$ relationship is impractical. It holds good only for heavy stratiform rain storms and changes significantly by the wind shear [24] during convective storms. In light rain, the attenuation is difficult to measure leading to large uncertainty in $R$. An advanced sensor like weather radar is capable of distinguishing rain intensities and storms easily by observing numerous target parameters (e.g. reflectivity, Doppler velocity, spectrum width and dual-polarization variables) [25]. However, satellite user terminals (UT) are single-polarized passive receivers that are unable to measure even the simplest parameters such as reflectivity and Doppler velocity that are necessary to classify rainfall intensity. Nearly all of existing mm-Wave weather radar algorithms have been developed for cloud observation in zenith-pointing mode effectively 
canceling the variation imparted by oblate raindrops [22]. On the other hand, the downlink rain estimates are directly affected by the raindrop canting angles [19]. Hence, applying existing mm-Wave radar rainfall procedures to UTs is not straightforward.

In this paper, we design a deep convolutional neural network (CNN) to overcome the aforementioned challenges of weather estimation using satellite downlinks. The input of the $\mathrm{CNN}$ are several profiles of specific attenuation from the specific UT location. The output is the rain intensity class obtained from a colocated radar or rain gauge. The proposed deep network then learns this relationship during the training stage. In the prediction stage, when the CNN operates online, the rain intensities are estimated by simply feeding the CNN with the downlink attenuation profiles so that the linear $R$ - $A$ relationship can be appropriately exploited. In the face of non-availability of critical meteorological observables, deep learning (DL) methods have the potential to extrapolate new features from a limited set of features contained in a training set [26]. In the past, S-, C-, X-, and $\mathrm{Ku}$-band weather radars have been shown to successfully use shallow networks for estimating rainfall (see e.g. [8] for an overview; [27, 28]) after training with rain gauge estimates. However, these techniques have traditionally relied on abundant auxiliary information such as dual-polarization, high sampling rates and low attenuation bands.

Contrary to these works, we use DL for satellite communication signals and retrieve rainfall in a more information-limited situation. Our previous work [9] showed the potential of Ka-band link for rainfall estimation where shallow networkrs were employed to classify only dry and wet conditions. In this work, we enhance the retrieval performance by training the ANN to classify other rain regimes using data from the nearest weather radar and colocated rain gauges. We show that our rain accumulations are in better agreement with the rain gauge than those from the nearest German weather service Deutscher Wetterdienst (DWD) radar.

The paper is organized as follows. In the next section, we provide the signal model and follow it with the details of the link setup in Section 3. In Section 4, we describe the DL-based technique to classify the precipitation intensities and present the performance evaluation with rain gauge and weather radar. We conclude in Section 5.

\section{SIGNAL MODEL}

Consider a radio-frequency (RF) emitter that sends a pulsed waveform $u_{t r}(t)$ through the rain medium. The precipitation target is volumetric and the signal received from a rain volume is the composite of echoes from many individual scatterers. These individual echoes constructively or destructively to produce a complex composite phasor sample. The received voltage is [29]

$$
V_{r}(t)=\sum_{k} A_{k}(\tau ; t) e^{-j 2 \pi f_{0} \tau_{k}} u_{t r}\left(t-\tau_{k}\right),
$$

where $A_{k}$ is the scattering amplitude of the $k$ th particle at range $r_{k}=c \tau_{k} / 2, \tau_{k}$ is the time delay associated with the range of the target, $f_{0}=c / \lambda$ is the carrier frequency of the radar, $c$ is the speed of light. The range $r_{k}$ of the independent scatterer being random, $V_{r}(t)$ is a complex sum of independent random variables. The central limit theorem [30] applies because, for the hydrometeor echoes, the number of scatterers is large and none of the variables is dominant. Therefore, the samples of $V_{r}$ form a Gaussian distribution with zero mean. If $V_{1}, V_{2}, \cdots, V_{N}$ form a set of $N$ complex received voltage samples corresponding to $\mathrm{N}$ consecutive transmitted pulses, then the probability density function of the signal vector $\mathbf{V}=\left[\begin{array}{llll}V_{1} & V_{2} & \cdots & V_{N}\end{array}\right]$ is a circular or proper [31] complex Gaussian distribution with zero mean,

$$
p(\mathbf{V})=\frac{1}{\pi^{N}\left|\mathbf{R}_{v v}\right|} \exp \left(-\mathbf{V}^{H} \mathbf{R}_{v v}^{-1} \mathbf{V}\right)
$$

where $\mathbf{R}_{v v}$ is the $N \times N$ complex covariance matrix corresponding to the signal vector $\mathbf{V}$.

The Probert-Jones transmission equation [32] for precipitation scattering of an RF signal relates the average received power $\bar{P}_{r}(\mathrm{~W})$ to the transmitted power $P_{t}(\mathrm{~W})$ as a function of the range $r_{0}(\mathrm{~m})$ of the scatterer:

$$
\bar{P}_{r}\left(r_{0}\right)=\left(\frac{c T_{0}}{2}\right)\left[\frac{P_{t} G_{0}^{2}}{\lambda^{2}(4 \pi)^{3}}\right]\left[\frac{\pi \theta_{1} \phi_{1}}{8 \ln 2}\right] \frac{\pi^{5}\left|K_{p}\right|^{2} Z_{e}\left(r_{0}\right)}{r_{0}^{2}}
$$

where $G_{0}$ denotes the peak antenna gain (dimensionless), $\theta_{1}$ and $\phi_{1}$ are the conventional half-power beam widths (rad), $T_{0}$ is the pulse width $(\mathrm{m})$ and $\lambda$ is the wavelength of the radar $(\mathrm{m}) . K_{p}$ is the complex dielectric factor (dimensionless) of the target (conventionally, water) so that $\left|K_{p}\right|^{2}=\left|\left(\varepsilon_{r}-1\right) /\left(\varepsilon_{r}+2\right)\right|^{2}$ where $\varepsilon_{r}$ is the relative permittivity. The $Z_{e}$ is the equivalent reflectivity factor $\left(\mathrm{mm}^{6} \mathrm{~m}^{-3}\right)$

$$
Z_{e}\left(r_{0}\right)=\frac{\lambda^{4}}{\pi^{5}\left|K_{p}\right|^{2}} \eta\left(r_{0}\right)
$$

where $\eta$ is the back-scatter cross-section per unit volume $\left(\mathrm{m}^{2} \mathrm{~m}^{-3}\right)$. In order to incorporate the effect of signal attenuation in (3), $Z_{e}$ is multiplied by the specific attenuation $A(\mathrm{~dB} / \mathrm{km})$. The equivalent reflectivity factor $Z_{e}$ is, by definition, proportional to the 6th moment of the raindrop diameter:

$$
Z_{e}=\int_{0}^{\infty} N(D) D^{6} d D
$$

where $N(D)\left(\mathrm{mm}^{-1} \mathrm{~m}^{-3}\right)$ denotes the raindrop size distribution (DSD) in a unit volume $\left(\mathrm{m}^{3}\right)$ and $D$ is the diameter of the raindrop (mm).

The rainfall rate $\mathrm{R}(\mathrm{mm} / \mathrm{h})$ being proportional the raindrop size, forms an empirical relationship with $Z_{e}$, and, in turn, the specific attenuation $A$. The measurement of the $A$ is, therefore, key to extract provide useful meteorological information. Specifically, at mm-Wave, the ITU recommends $[33,34]$ the following power-law relationship

$$
A=k R^{\alpha},
$$

where the coefficients $k$ and $\alpha$ depend on the frequency and polarization of the link. In this paper, for the experimental setup at forward link, we compute these values as $k=0.0939$ and $\alpha=1.0199$. The specific attenuation is obtained as $A=D / L_{s}$ where $D$ is the absolute signal attenuation and $L_{s}=4.9276 \mathrm{~km}$ is the slant path length of the satellite link [9].

\section{EXPERIMENTAL SETUP}

The broadband satellite systems offer bidirectional communications between the gateway stations and UTs to enable interactive satellite services (such as Internet provisioning) (Fig. 1). The link from gateway to UTs is called the forward/downlink and the link from UTs to gateway is the return/uplink. In order to maintain a certain level of quality of service for users, GW stations continuously monitor the up and downlinks. The carrier-to-noise ratio $(C / N)$ parameter measures the quality of the communication link and received signals. The gateway has access to end-to-end $C / N$ on both links. However, we use only forward links $C / N$ for rainfall estimation due to 


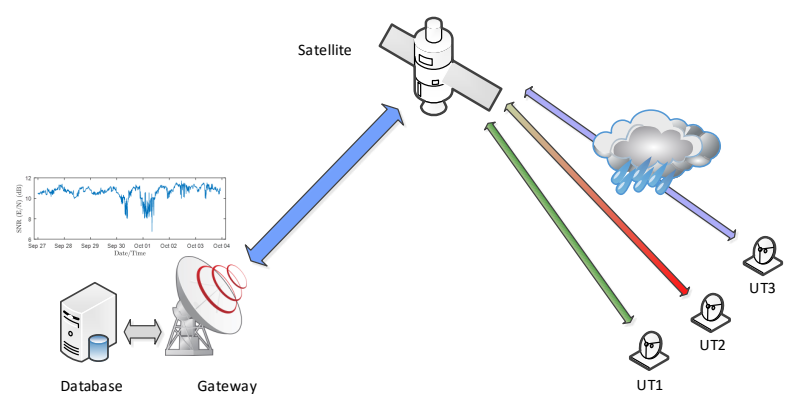

Fig. 1. Illustration of broadband satellite communication systems.

limitations in accessing return link data. Average $C / N$ is available at the gateway station every five minutes (average $C / N$ over five minutes) and stored in a database. It is generally assumed that the link between GW and the satellite (feeder link) is ideal. This is due to the fact that the transmit/receive antenna gain of the $\mathrm{GW}$ is very high Therefore, the end-to-end $C / N$ is dominated by the $C / N$ of the user link (links between satellite and UTs). As a result, it can be assumed that variations of the end-to-end $C / N$ are mainly caused by the user links. The $C / N$ parameter is mainly affected by the rain attenuation at the operational Ka-band frequencies (broadband satellite) [35]. Therefore, there is a clear correlation between the $C / N$ variations and amount of the rainfall.

In order to illustrate our proposed method, we have exploited the experimental system installed in Betzdorf, Luxembourg. The system includes a broadband satellite UT and a weather station equipped with rain gauge. The UT receives broadband services from ASTRA $2 \mathrm{~F}$ satellite located at the orbital position of $28.2 \mathrm{E}$. The links from satellite to UT operate at K-Band (19.70-20.20 GHz) while the links from UT to satellite is at Ka-Band $(29.40-30.00 \mathrm{GHz})$. The elevation and azimuth angles of the links are $29.4^{\circ}$ and $151.8^{\circ}$, respectively. We also used rainfall data from the C-band DWD weather radar (located in Southwest Germany) for studying the rain events over the satellite link. Fig. 2 depicts the relative locations of our set-up.

After training the rainfall estimation algorithm using the experimental set-up in Beztdorf, we applied it to $C / N$ data of 35 UTs. These UTs are distributed in $25 \mathrm{~km} \times 20 \mathrm{~km}$ area in Southwest Germany. Fig. 3 shows the distribution and location of the 35 UTs in this region.

The first step towards estimating the rainfall from $C / N$ measurements is to determine the amount of signal attenuation caused by rainfall during the rain event. Central to this, is an understanding of the various components constituting the $C / N$ and the impact of rainfall on them. To this end, let us denote $C / N$ by $C(n)$, where $n$ is the index of measurement (data sample). Note that the data indexing starts from midnight and has a temporal resolution of 5 minutes, i.e., $n=2$ represents 0005 UTC time of the first day of data collection.

Radio-wave propagation (and $C / N$ ) on Earth-Space links in millimeter wave frequencies is impacted by different tropospheric effects [36], the major factor being the signal attenuation due to rain medium. We remark that co-channel interference also affects $C / N$. However, our set up does not employ multibeam coverage and frequency reuse. Consequently, the effect of co-channel interference is limited. Therefore, we assume that $C / N$ is only impacted by rain attenuation and can decompose $C / N$ (in $\mathrm{dB}$ ) as,

$$
C(n)=B(n)-D(n),
$$

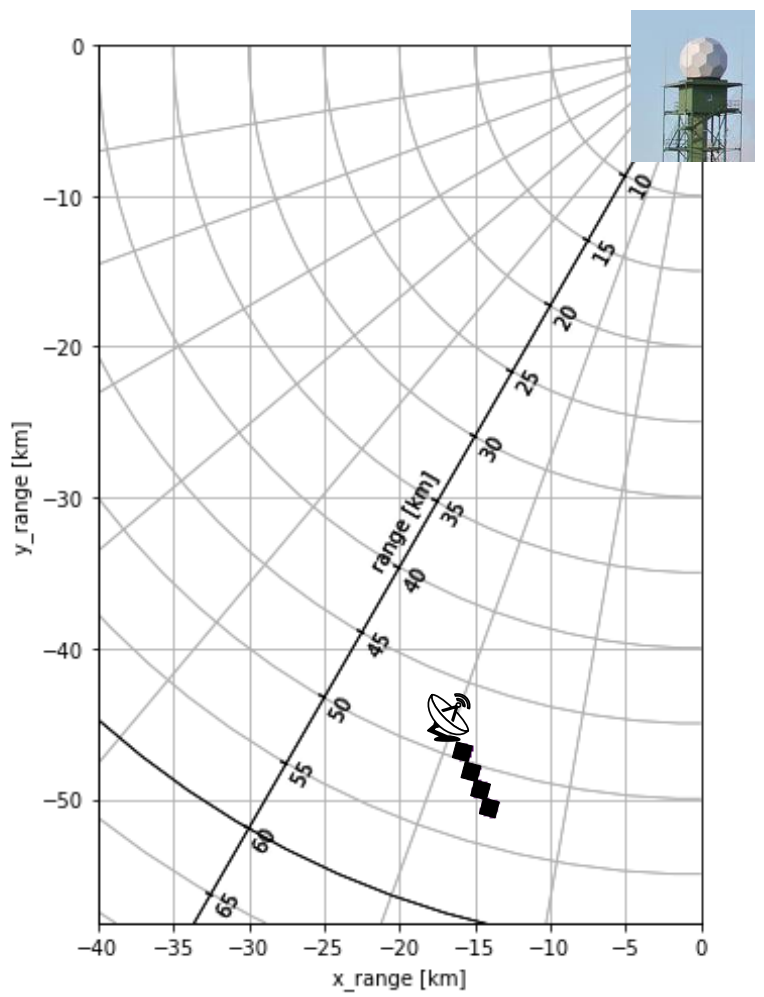

Fig. 2. Approximate projection of the satellite slant path on four radar bins shown by dark color. The first top left of these four black bins shows the location of the UT as well as the collocated rain gauge. The Southwest Germany DWD radar is about $50 \mathrm{~km}$ away from the UT.

where, $B(n)$ is the baseline (reference level) that corresponds to the expected $C / N$ in dry (no-rain) situation assuming a fixed position. The $B(n)$ includes impact of gaseous absorption, cloud attenuation, scintillations and noise in the microwave link. The $D(n)$ accounts for attenuation in rain and is zero in dry situations. As mentioned earlier, at any instant $n, A(n)=D(n) / L$ is the specific attenuation.

\section{ESTIMATION FROM DEEP CONVOLUTIONAL NETWORKS}

We now design a CNN which accepts a matrix of size $R=400$ realizations by $N=200$ time instances with corresponding $A$ values for each UT location. The proposed network (Fig. 4) is composed of ten layers. The first layer is the input layer; second and fourth layers are the convolutional layers with 512 filters of size $3 \times 3$ to extract the features hidden in the input data. After each convolutional layer, there is a normalization layer to normalize the output and provide better convergence. The sixth and eighth layers are fully connected layers with 1024 units, respectively. There are dropout layers after the fully connected layers (the seventh and ninth layers) with a $50 \%$ probability. The output is a three-class output layer which delineates rain storms as dry, light, and heavy.

In order to obtain the labels of the network, we trained the network on the nearest DWD radar and colocated rain-gauge data. All 


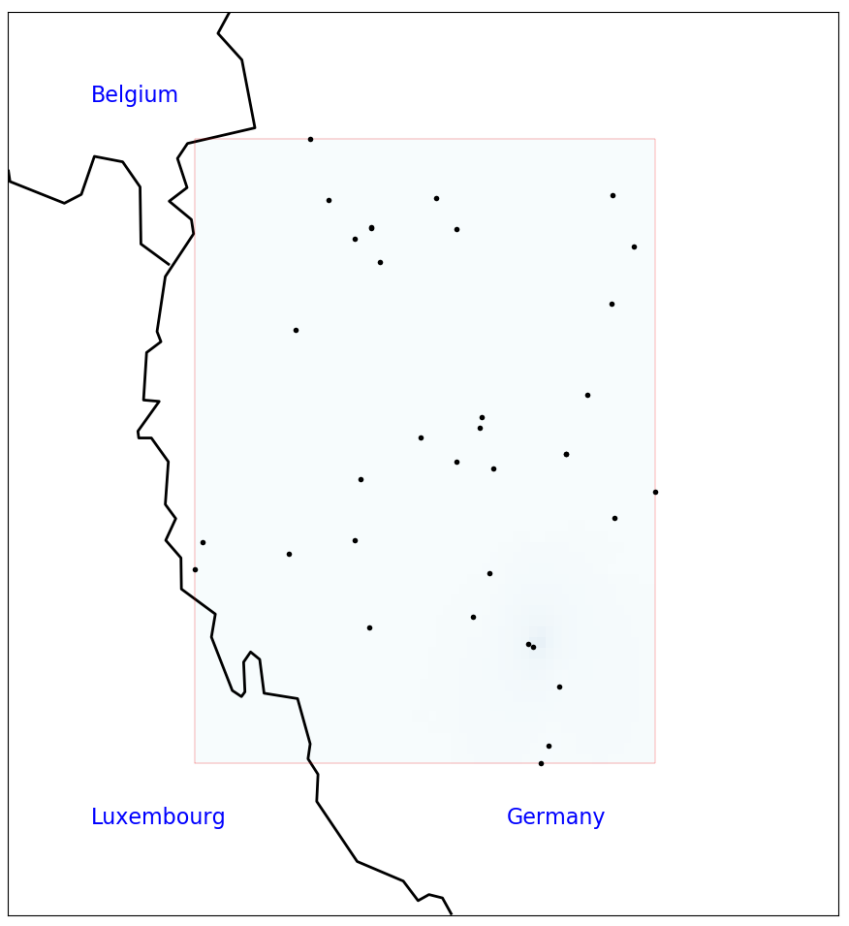

Fig. 3. Location of 35 satellite terminals in Southwest Germany (25km by $20 \mathrm{~km})$.

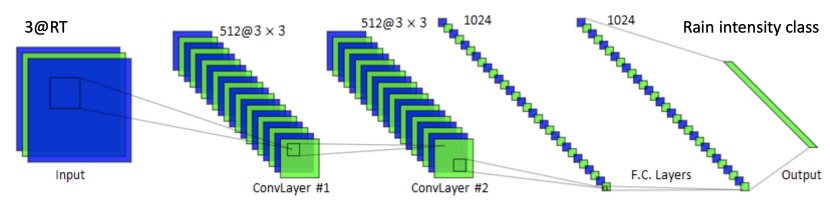

Fig. 4. The proposed network architecture for rain intensity classification.

nets were trained with gradient descent, using a learning rate of $10^{-1}$ and a momentum of 0.9. At the end of each iteration, the weights were updated and network activations were reset to zero. The output layers had softmax classifers, and the cross entropy objective function was used for training. The classification of rain regimes is required to find the baseline and signal attenuation $(B(n)$ and $D(n)$, respectively). We calculate the rain rate using $D(n)$ as recommended by the ITU-R model [33, 34] (see Section 2).

After identifying the rain and dry events, we use a procedure similar to the one discussed in [9] to estimate the signal attenuation due to rain, i.e., $D(n)$. Following the procedure outlined in Section 2, we obtained estimates of $R$ for each UT. Fig. 5 shows rain rates and rain accumulations over 24 hour period from 1200 UTC on 22 May 2018 to 1200 UTC on 23 May 2018 as estimated by the Southwest Germany DWD radar (dashed blue line) and the satellite link UT (encircled terminal on Fig. 3) using our proposed algorithm (solid red line). The UT estimates follows the radar closely leading to a single-day accumulation difference of $\sim 1.5 \mathrm{~mm}$ only.

\section{SUMMARY}

We proposed and investigated a DL framework for rainfall estimation based on opportunistic use of $C / N$ measurements from broadband
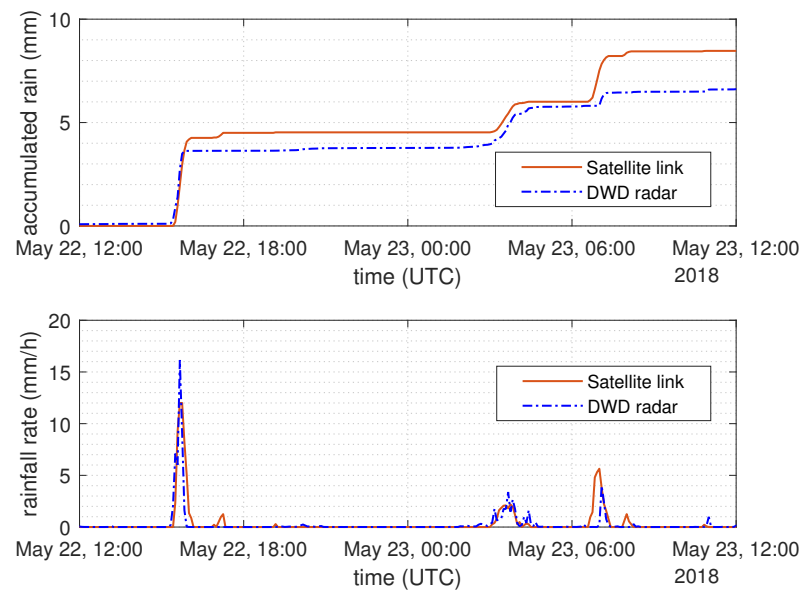

Fig. 5. Rain accumulations during 24 hours from 1200 UTC on 22 May 2018 till 1200 UTC on 23 May 2018 as observed by the Southwest Germany DWD radar (dashed blue line) and satellite link (encircled UT in Fig. 3) using our proposed algorithm (solid red line).

satellite communication networks. The learning network was modeled after $\mathrm{CNN}$ which searches and recognizes long-term temporal dependencies in time-series data. We trained CNN to directly classify the $C / N$ data from the satellite links into dry and wet weather without using any user-defined data features. Thereafter, the events classified as rain storms were estimated for rain rates using the $R-A$ expression following the ITU-R recommendations. Our comparisons of the rainfall distribution map generated from CNN-based UT estimates with the nearest weather radar shows that our algorithm captures the major features of the storm even though very limited information about the weather was available to the UT set-up. This study demonstrates that DL is a promising framework to extract valuable, although less accurate, precipitation retrievals via the communication satellite links. In future, our goal is to continue improving the UT estimates through use of other advanced DL techniques and better training data.

\section{REFERENCES}

[1] I. Mallakpour and G. Villarini, "The changing nature of flooding across the central United States," Nature Climate Change, vol. 5, no. 3, p. 250, 2015.

[2] L. Yang, J. A. Smith, D. B. Wright, M. L. Baeck, G. Villarini, F. Tian, and $\mathrm{H}$. Hu, "Urbanization and climate change: An examination of nonstationarities in urban flooding," Journal of Hydrometeorology, vol. 14, no. 6, pp. 1791-1809, 2013.

[3] K. V. Mishra, W. F. Krajewski, R. Goska, D. Ceynar, B.-C. Seo, A. Kruger, J. J. Niemeier, M. B. Galvez, M. Thurai, V. Bringi et al., "Deployment and performance analyses of highresolution Iowa XPOL radar system during the NASA IFloodS campaign," Journal of Hydrometeorology, vol. 17, no. 2, pp. 455-479, 2016.

[4] W. Krajewski and J. Smith, "Radar hydrology: Rainfall estimation," Advances in Water Resources, vol. 25, no. 8-12, pp. 1387-1394, 2002.

[5] G. Villarini and W. F. Krajewski, "Review of the different sources of uncertainty in single polarization radar-based es- 
timates of rainfall," Surveys in Geophysics, vol. 31, no. 1, pp. 107-129, 2010.

[6] B.-C. Seo, W. F. Krajewski, and K. V. Mishra, "Using the new dual-polarimetric capability of WSR-88D to eliminate anomalous propagation and wind turbine effects in radar-rainfall," Atmospheric Research, vol. 153, pp. 296-309, 2015.

[7] M. Thurai, K. V. Mishra, V. Bringi, and W. F. Krajewski, "Initial results of a new composite-weighted algorithm for dualpolarized X-band rainfall estimation," Journal of Hydrometeorology, vol. 18, no. 4, pp. 1081-1100, 2017.

[8] F. Y. Testik and M. Gebremichael, Rainfall: State of the science. John Wiley \& Sons, 2013, vol. 191.

[9] A. Gharanjik, M. R. Bhavani Shankar, F. Zimmer, and B. Ottersten, "Centralized rainfall estimation using carrier-to-noise of satellite communication links," IEEE Journal on Selected Areas in Communications, 2018, in press.

[10] L. Barthès and C. Mallet, "Rainfall measurement from opportunistic use of earth-space link in Ku band," Atmospheric Measurement Techniques, vol. 6, no. 8, pp. 2181-2193, 2013.

[11] C. Mugnai, F. Sermi, F. Cuccoli, and L. Facheris, "Rainfall estimation with a commercial tool for satellite internet in Ka band: Model evolution and results," in IEEE International Geoscience and Remote Sensing Symposium, 2015, pp. 890-893.

[12] A. Gharanjik, K. V. Mishra, M. R. Bhavani Shankar, and B. Ottersten, "Learning-based rainfall estimation via communication satellite links," in IEEE Statistical Signal Processing Workshop, 2018.

[13] A. Y. Hou, G. Skofronick-Jackson, C. D. Kummerow, and J. M. Shepherd, "Global precipitation measurement," in Precipitation: Advances in Measurement, Estimation and Prediction, S. C. Michaelides, Ed. Springer, 2008.

[14] J. Ostrometzky and H. Messer, "Dynamic determination of the baseline level in microwave links for rain monitoring from minimum attenuation values," IEEE Journal of Selected Topics in Applied Earth Observations and Remote Sensing, vol. 11, no. 1, pp. 24-33, 2018.

[15] K. V. Mishra, V. Chandrasekar, C. Nguyen, and M. Vega, "The signal processor system for the NASA dual-frequency dualpolarized Doppler radar," in IEEE International Geoscience and Remote Sensing Symposium, 2012, pp. 4774-4777.

[16] S. Seto and T. Iguchi, "Intercomparison of attenuation correction methods for the GPM dual-frequency precipitation radar," Journal of Atmospheric and Oceanic Technology, vol. 32, no. 5, pp. 915-926, 2015.

[17] K. V. Beard, V. Bringi, and M. Thurai, "A new understanding of raindrop shape," Atmospheric Research, vol. 97, no. 4, pp. 396-415, 2010.

[18] K. Nakamura, Y. Kaneko, K. Nakagawa, H. Hanado, and M. Nishikawa, "Measurement method for specific attenuation in the melting layer using a dual Ka-band radar system," IEEE Transactions on Geoscience and Remote Sensing, vol. 56, no. 6, pp. 3511-3519, 2018.

[19] V. Bringi, M. Thurai, and D. Brunkow, "Measurements and inferences of raindrop canting angles," Electronics Letters, vol. 44, no. 24, pp. 1425-1426, 2008.
[20] S. Y. Matrosov, "Attenuation-based estimates of rainfall rates aloft with vertically pointing Ka-band radars," Journal of Atmospheric and Oceanic Technology, vol. 22, no. 1, pp. 43-54, 2005.

[21] R. Lhermitte, "A 94 GHz Doppler radar for clouds observations," Journal of Atmospheric and Oceanic Technology, vol. 4, pp. 3648, 1987.

[22] A. Chandra, C. Zhang, P. Kollias, S. Matrosov, and W. Szyrmer, "Automated rain rate estimates using the Ka-band ARM zenith radar (KAZR)," Atmospheric Measurement Techniques, vol. 8, no. 9, p. 3685, 2015.

[23] S. Y. Matrosov, P. T. May, and M. D. Shupe, "Rainfall profiling using atmospheric radiation measurement program vertically pointing 8-mm wavelength radars," Journal of Atmospheric and Oceanic Technology, vol. 23, no. 11, pp. 1478-1491, 2006.

[24] M. P. Mittermaier, R. J. Hogan, and A. J. Illingworth, "Using mesoscale model winds for correcting wind-drift errors in radar estimates of surface rainfall," Quarterly Journal of the Royal Meteorological Society, vol. 130, no. 601, pp. 2105-2123, 2004.

[25] B. Geerts and Y. Dawei, "Classification and characterization of tropical precipitation based on high-resolution airborne vertical incidence radar. Part I: Classification,' Journal of Applied Meteorology, vol. 43, no. 11, pp. 1554-1566, 2004.

[26] Y. Lecun, Y. Bengio, and G. Hinton, "Deep learning," Nature, vol. 521, no. 7553, pp. 436-444, 2015.

[27] G. Vulpiani, S. Giangrande, and F. S. Marzano, "Rainfall estimation from polarimetric S-band radar measurements: Validation of a neural network approach," Journal of Applied Meteorology and Climatology, vol. 48, no. 10, pp. 2022-2036, 2009.

[28] S. Orlandini and I. Morlini, "Artificial neural network estimation of rainfall intensity from radar observations," Journal of Geophysical Research: Atmospheres, vol. 105, no. D20, pp. 24 849-24 861, 2000.

[29] R. J. Doviak and D. S. Zrnić, Doppler Radar and Weather Observations. Dover Publications, Inc., 1993.

[30] A. Papoulis and S. U. Pillai, Probability, Random Variables and Stochastic Processes, 4th ed. McGraw-Hill, 2002.

[31] P. J. Scherier and L. L. Scharf, Statistical Signal Processing of Complex-Valued Data: The Theory of Improper and Noncircular Signals. Cambridge University Press, 2010.

[32] J. R. Probert-Jones, "The radar equation in meteorology," Quarterly Journal of Royal Meteorological Society, vol. 88, pp. 485495, 1962.

[33] Specific attenuation model for rain for use in prediction methods, Geneva, Switzerland, 2005.

[34] Propagation data and prediction method required for the design of the Earth-space telecommunication systems, Geneva, Switzerland, 2013.

[35] A. Gharanjik, M. R. Bhavani Shankar, P.-D. Arapoglou, and B. Ottersten, "Multiple gateway transmit diversity in $\mathrm{Q} / \mathrm{V}$ band feeder links," IEEE Transactions on Communications, vol. 63, no. 3, pp. 916-926, 2015.

[36] A. D. Panagopoulos, P.-D. M. Arapoglou, and P. G. Cottis, "Satellite communications at $\mathrm{Ku}, \mathrm{Ka}$, and V bands: Propagation impairments and mitigation techniques," IEEE Communications Surveys and Tutorials, vol. 6, no. 3, pp. 2-14, 2004. 